\title{
Photoacoustic imaging of cancer cells with glycol-chitosan-coated gold nanoparticles as contrast agents
}

\author{
In-Cheol Sun \\ Cheol-Hee Ahn \\ Kwangmeyung Kim \\ Stanislav Emelianov
}




\title{
Photoacoustic imaging of cancer cells with glycol-chitosan-coated gold nanoparticles as contrast agents
}

\author{
In-Cheol Sun, ${ }^{a, b}$ Cheol-Hee Ahn, ${ }^{c}$ Kwangmeyung Kim, ${ }^{d}$ and Stanislav Emelianov ${ }^{a, b, *}$ \\ ${ }^{a}$ Georgia Institute of Technology, School of Electrical and Computer Engineering Atlanta, Georgia, United States \\ ${ }^{b}$ Georgia Institute of Technology and Emory University School of Medicine, Wallace H. Coulter Department of Biomedical Engineering, \\ Atlanta, Georgia, United States \\ 'Seoul National University, NanoBio Materials Laboratory, Department of Materials Science and Engineering, Seoul, Republic of Korea \\ ${ }^{d}$ Korea Institute of Science and Technology, Biomedical Research Institute, Seoul, Republic of Korea
}

\begin{abstract}
Utility of glycol-chitosan-coated gold nanoparticles (GC-AuNPs) as a photoacoustic contrast agent for cancer cell imaging was demonstrated. Through the synergistic effect of glycol chitosan and gold nanoparticles, GC-AuNPs showed cellular uptake in breast cancer cells and resulted in strong photoacoustic signals in tissue-mimicking cell phantoms. The performance of GC-AuNPs as contrast agents was established with photoacoustic imaging and confirmed with dark-field microscopy. The cell phantoms displayed strong photoacoustic signals if cells were incubated more than $3 \mathrm{~h}$ with GC-AuNPs, compared with PEG-AuNPs that showed no photoacoustic signal increase. The enhanced photoacoustic signals originated from the plasmon coupling effect of GC-AuNPs after the cellular uptake in cancer cells. Importantly, photoacoustic imaging of cancer cells was achieved with GC-AuNPs - contrast agents that did not require antibodies or complex surface modification. The endocytosis of GC-AuNPs was also confirmed with dark-field microscopy. The results show that GC-AuNPs have potential as a photoacoustic contrast agent for cellular imaging including tumor tissue imaging. ( ) The Authors. Published by SPIE under a Creative Commons Attribution 4.0 Unported License. Distribution or reproduction of this work in whole or in part requires full attribution of the original publication, including its DOI. [DOI: 10.1117/1.JBO.24.12.121903]
\end{abstract}

Keywords: glycol chitosan; gold nanoparticles; photoacoustic imaging; cancer imaging; contrast agent.

Paper 190001LR received Jan. 3, 2019; accepted for publication Jul. 11, 2019; published online Aug. 5, 2019.

\section{Introduction}

Ultrasound imaging is a safe and cost-effective imaging modality capable of high spatial and temporal resolutions, and penetration depth. Nonetheless, applications of ultrasound imaging are often limited by low contrast because the difference of acoustic impedance between healthy and diseased tissues is often small. ${ }^{1}$ Consequently, there are several approaches to improve contrast in ultrasound imaging, for example, imaging using ultrasound contrast agents, ${ }^{2}$ ultrasound elasticity imaging, ${ }^{3}$ or a combination of ultrasound imaging with other synergistic imaging modalities, such as photoacoustic imaging. ${ }^{4}$

Photoacoustic imaging provides high-contrast images by using endogenous or exogenous chromophores with distinct optical absorption property. ${ }^{5-7}$ If the exogenous contrast agent is designed to absorb pulsed laser light of specific wavelengths that is away from the optical absorption of endogenous tissue chromophores, then generated photoacoustic signals have minimal overlap with background tissue signals. ${ }^{8}$

Among various contrast agents, gold nanoparticles (AuNPs) are promising exogenous contrast agents because they have unique optical properties that are desirable for photoacoustic imaging. ${ }^{8}$ Upon exposure to laser irradiation, AuNPs absorb light through a surface plasmon resonance (SPR) phenomenon. Specifically, the absorption of AuNPs is the highest if the frequency of incident light is similar to the resonance frequency of surface plasmon. This resonance phenomenon is called SPR, which results in the high absorption peak of AuNPs in the UV-vis-NIR spectrum.

\footnotetext{
*Address all correspondence to Stanislav Emelianov, E-mail: stas@ gatech.edu
}

The low thermal diffusivity of AuNPs is also an important factor that is a prerequisite for photoacoustic imaging. ${ }^{9,10}$

We introduce glycol-chitosan-coated gold nanoparticles (GC-AuNPs) as a photoacoustic contrast agent for cancer cell imaging. GC is a derivative of chitosan with ethylene glycol moieties, substituting hydroxyl groups of some polysaccharide repeating units for improved hydrophilicity. ${ }^{11} \mathrm{GC}$ has the potential to serve as a multipurpose imaging and therapeutic agent because amine groups in the repeating unit provide sites for chemical modification. Moreover, GC nanoparticles exhibit enhanced tumor accumulation. ${ }^{12,13}$ Due to enhanced stability in the body and for its tumor-targeting property, GC has been used as a coating material of AuNPs. ${ }^{14-16}$ Previously, GCAuNPs have been developed as contrast agents for several biomedical imaging modalities. For example, GC-AuNPs and fluorescent dyes were conjugated to visualize the activity of the matrix metalloproteinase enzyme in cancer. ${ }^{15}$ GC-AuNPs were also exploited as a computed tomography contrast agent for tumor imaging because of x-ray absorption properties of gold. ${ }^{16}$ Furthermore, the enhanced tumor-targeting of GC-AuNPs compared with the cells of the immune system such as macrophages was shown. ${ }^{16}$

In this paper, we report an application of GC-AuNPs as a photoacoustic agent for cancer cell imaging. Previously, the feasibility of AuNPs as a photoacoustic contrast agent was reported for adipose-derived stem-cell tracking. ${ }^{17}$ The aggregation of AuNPs inside stem cells after incubation induced a significant local temperature rise upon pulsed laser irradiation, which resulted in enhanced photoacoustic signals in stem cells. ${ }^{18}$ Similarly, antibody-targeted AuNPs were used to detect primary tumor ${ }^{19}$ or metastatic cancer cells in lymph nodes. ${ }^{20}$ 
We hypothesize that GC-AuNPs accumulate in cancer cells by the surface property of GC and produce strong photoacoustic signals after endocytosis. The characterization of GC-AuNPs and photoacoustic imaging of cancer cell phantoms prove the potential of GC-AuNPs as a contrast agent for cancer cell imaging.

\section{Methods}

GC-AuNPs were synthesized as previously reported. ${ }^{15}$ Briefly, GC ( $1 \mathrm{mg} / \mathrm{ml}$, degree of polymerization $\geq 400$, Sigma-Aldrich Corp., St. Louis, Missouri) solution was boiled to $70^{\circ} \mathrm{C}$ and mixed with $\mathrm{HAuCl}_{4} \cdot 3 \mathrm{H}_{2} \mathrm{O}$ solution $(1 \mathrm{mM}, 100 \mathrm{ml})$ under stirring for $24 \mathrm{~h}$ until the solution turned red. Afterward, the GC-AuNP colloid was washed twice through centrifugation $(10,000 \mathrm{rpm}$ for $50 \mathrm{~min}$ ) with distilled water and sonicated for less than 1 min before use. As a control, PEGylated AuNPs (PEG-AuNPs) were synthesized. First, citrate-reduced AuNPs were synthesized by adding $0.05 \mathrm{wt} . \%$ of sodium citrate in the boiling $\mathrm{HAuCl}_{4} \cdot 3 \mathrm{H}_{2} \mathrm{O}$ solution $(1 \mathrm{mM}, 100 \mathrm{ml})$ under stirring. After 30 min, citrate-reduced AuNPs colloid was cooled down to room temperature and washed twice with a centrifuge (10,000 rpm for $30 \mathrm{~min}$ ). For PEGylation of citrate-reduced AuNPs, 1 wt.\% of mPEG-SH (Laysan Bio Inc., Arab, Alabama) was dissolved in the AuNPs colloid and kept under stirring for $24 \mathrm{~h}$. Before use, excessive PEG molecules were removed by centrifuge $(10,000 \mathrm{rpm}$ for $30 \mathrm{~min})$ and washed twice with distilled water.

The absorption spectra of GC-AuNPs, citrate-reduced AuNPs, and PEG-AuNPs were measured from 350 to $850 \mathrm{~nm}$ in a microplate reader (Synergy ${ }^{\mathrm{TM}} \mathrm{HT}$, BioTek Instruments, Winooski, Vermont). Transmission electron microscopic (TEM) images of the GC-AuNPs and PEG-AuNPs were taken in JEOL 2010-F TEM operating at $200 \mathrm{kV}$. TEM samples were prepared by dropping $10 \mu \mathrm{l}$ of a nanoparticle colloid onto carbon-coated, 200-mesh copper grids. The particle size distribution was obtained in Zetasizer Nano ZS (Malvern Instruments, Malvern, UK), in which the intensity-weighted mean diameter was calculated by dynamic light scattering (DLS).

MDA-MB-231 breast cancer cells were cultured in Dulbecco's modified Eagle medium media containing fetal bovine serum $(10 \%)$, penicillin $(100 \mathrm{U} / \mathrm{ml})$, and streptomycin $(100 \mathrm{mg} / \mathrm{ml})$ until their confluence reached $70 \%$ to $80 \%$ in $100-\mathrm{mm}$ culture dishes. The cells were seeded at a density of $2 \times 10^{4}$ cells on a gelatin-coated coverslip in six-well plates. For cellular uptake, the cells were incubated in the culture medium containing either PEG-AuNPs or GC-AuNPs (0.05 mg Au/ml) for $1,2,3$, and $4 \mathrm{~h}$, then washed twice with PBS ( $\mathrm{pH} 7.4$ ), detached with trypsin/ethylenediaminetetraacetic acid, and fixed with paraformaldehyde solution (4\%) for $10 \mathrm{~min}$ at room temperature. For bright- and dark-field microscopy, the coverslips, on which the cells were incubated with either PEG-AuNPs or GC-AuNPs, were mounted on a glass slide and imaged using a Leica DMI3000B microscope (Leica Microsystems, Wetzlar, Germany). For photoacoustic imaging of cells, the phantoms with three-dimensional tissue models were prepared as reported previously. ${ }^{21}$ To prepare the phantom base, type-A 300-Bloom gelatin derived from acid-cured porcine skin (15 g, Sigma-Aldrich Corp.) was mixed with distilled water $(250 \mathrm{ml})$ containing $0.1 \%$ (wt./wt.) formaldehyde solution (37\% wt./wt., Thermo-Fisher Scientific Inc., Waltham, Massachusetts) under vigorous stirring. Then, $40-\mu \mathrm{m}$ silica particles (0.75 g, U.S. Silica Co., Mill Creek, Oklahoma) were added to the gelatin solution for enhanced ultrasonic backscattering. The mixture was slowly heated to $45^{\circ} \mathrm{C}$ and placed in a vacuum chamber at $21 \mathrm{kPA}$ pressure for $10 \mathrm{~min}$ to remove bubbles entrapped in the mixture. Afterward, the mixture was poured into a cuvette and cooled down in a $4^{\circ} \mathrm{C}$ refrigerator for $12 \mathrm{~h}$, thus creating a phantom base on which the 3-D tissue models were placed. To prepare cell inclusions, a second solution of gelatin was prepared. The cells, previously incubated with nanoparticles for 1, 2, 3, or $4 \mathrm{~h}$, washed twice with PBS and fixed in paraformaldehyde solution, were mixed with gelatin solution at $40^{\circ} \mathrm{C}$ and pipetted onto the phantom base to form approximately $40-\mu 1$ dome-shaped semispherical inclusions measuring $4 \times 4 \times 2 \mathrm{~mm}^{3}\left(\sim 15 \mathrm{~mm}^{3}\right)$ The cell concentration in each inclusion was $1.8 \times 10^{4} \mathrm{cell} / \mu \mathrm{l}$. Overall, four phantoms (1, 2, 3, and $4 \mathrm{~h}$ incubation) with two inclusions (GC-AuNPs and PEG-AuNPS) in each phantom were prepared.

The phantoms were imaged using a Vevo ${ }^{\circledR}$ LAZR imaging system (VisualSonics, Toronto, Canada) and a 20-MHz US array transducer interfaced with fiber optic light delivery (LZ250, VisualSonics). The ultrasound-guided photoacoustic imaging was performed at 5 frames/s using Q-switched Nd:YAG laser pumped OPO system (680 to $960 \mathrm{~nm}$ wavelength range, 7-ns laser pulse duration). Data were exported and postprocessed in MATLAB (MathWorks, Inc. Natick, Massachusetts).

\section{Results}

GC-AuNPs were characterized based on UV-vis-NIR absorption spectrum, TEM images, and DLS measurements. The SPR peak of GC-AuNPs was at $528 \mathrm{~nm}$, while that of citrate-reduced AuNPs and PEG-AuNPs with similar size was at 520 and $523 \mathrm{~nm}$, respectively [Fig. 1(a)]. The TEM images revealed the spherical morphology and no aggregation of GC-AuNPs
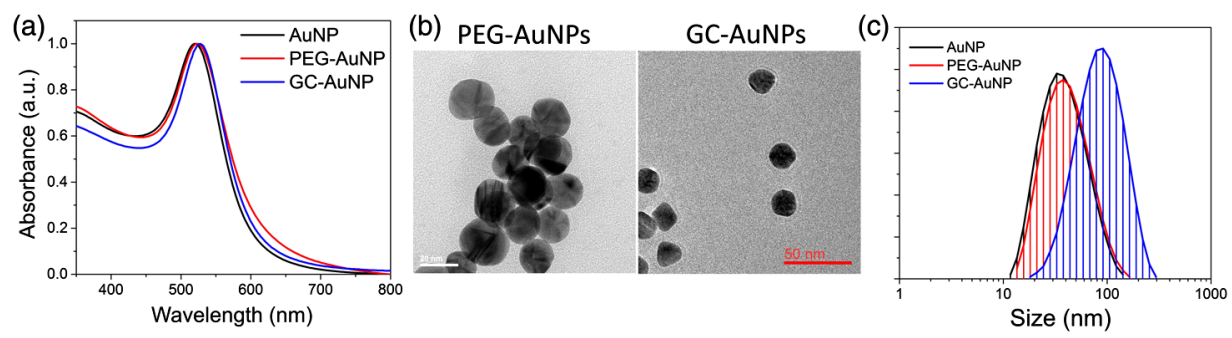

Fig. 1 (a) UV-vis spectra of citrate-stabilized AuNPs, PEG-AuNPs, and GC-AuNPs show the red-shift of SPR peak. Specifically, SPR peak of GC-AuNPs is at $528 \mathrm{~nm}$ while that of AuNPs and PEG-AuNPs are at 520 and $523 \mathrm{~nm}$, respectively. (b) TEM images indicate the spherical morphology of both PEG-AuNPs and GC-AuNPs. Coating layer can be observed around GC-AuNPs. (c) Hydrodynamic sizes of AuNPs, PEGAuNPs, and GC-AuNPS are $17.89 \pm 5.47 \mathrm{~nm}, 27.84 \pm 12.06 \mathrm{~nm}$, and $94.46 \pm 46.45$, respectively. 

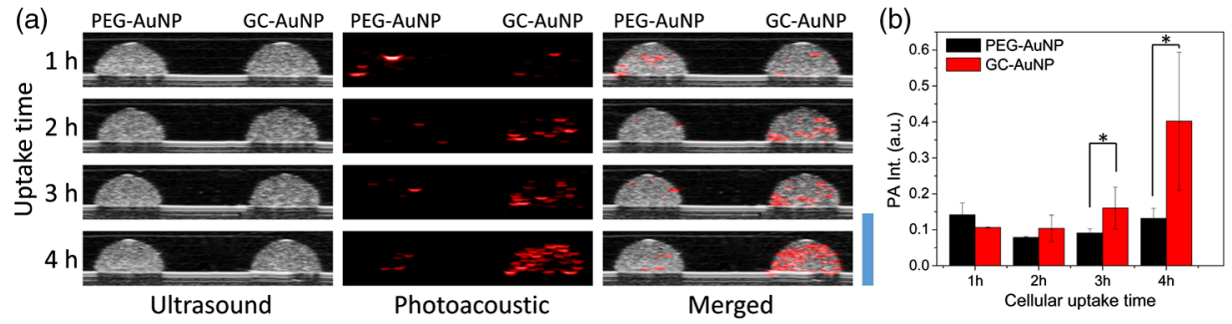

Fig. 2 (a) Ultrasound (left column), photoacoustic (middle column), and merged (right column) images of inclusions containing MDA-MB-231 cells incubated in cell culture media containing AuNPs for 1, 2, 3, and $4 \mathrm{~h}$, respectively. GC-AuNPs were taken up by cells (right-hand side inclusion in each image); PEG-AuNPs were used in the (left) phantom as a control. Each cell phantom contained $3.6 \times 10^{5}$ cells. In photoacoustic images, the laser wavelength was $680 \mathrm{~nm}$. Scale bar is $4 \mathrm{~mm}$. (b) Significant increase in photoacoustic signal from cells incubated with GC-AuNPs appears after $3 \mathrm{~h}$ of cellular uptake, compared with PEG-AuNPs [t-test, $n=3$ for each group, $p=0.01393(3 \mathrm{~h})$, and $p=0.012384(4 \mathrm{~h})$ ].

[Fig. 1(b)]. In particular, a thin coating layer is noticeable around the GC-AuNP surface. The hydrodynamic size of GC-AuNPs was $94.46 \pm 46.45 \mathrm{~nm}$ while AuNPs and PEG-AuNPs were $17.89 \pm 5.47 \mathrm{~nm}$ and $27.84 \pm 12.06 \mathrm{~nm}$, respectively [Fig. 1(c)]. The size of GC-AuNPs was different from citrate-coated AuNPs or PEG-AuNPs because of hydrophilicity of GC coating layer. The GC coating also affected the surface property of GC-AuNPs. The zeta-potential value of GC-AuNPs was $37.4 \pm 4.4 \mathrm{mV}$, while AuNPs and PEG-AuNPs were $-29.2 \pm 4.9 \mathrm{mV}$ and $-3.28 \pm 0.75 \mathrm{mV} \mathrm{mV}$, respectively.

Ultrasound and photoacoustic images of tissue-mimicking cell phantoms are presented in Fig. 2. Cell inclusions showed strong photoacoustic signals if cells were incubated with GCAuNPs more than $3 \mathrm{~h}$ [Fig. 2(a)]. The intensity of photoacoustic signals increased as cellular uptake time increased [Fig. 2(b)]. In contrast, photoacoustic signals from cancer cell incubated with PEG-AuNPs were inconspicuous even after $4 \mathrm{~h}$ of incubation. Photoacoustic signals detected from the cell phantoms of PEG-AuNPs were regarded as noise because no specific spatial patterns of signal changes were observed. The significant differences between GC-AuNPs and PEG-AuNPs were also described by an unpaired $t$-test, in which the $p$-values of 3 and $4 \mathrm{~h}$ of incubation were 0.0139 and 0.0124 , respectively [Fig. 2(b)].

The magnitude of photoacoustic signals from cell phantoms was measured within 680 to $960 \mathrm{~nm}$ wavelength range to demonstrate the wavelength-dependent intensity of the photoacoustic signal. The photoacoustic response from GC-AuNP cells was the strongest at $680 \mathrm{~nm}$ and then gradually decreased as the wavelength of the laser irradiation increased (Fig. 3). In contrast, cancer cells incubated with PEG-AuNPs show low amplitude and nearly constant photoacoustic signals regardless of the laser wavelength. These measurements are consistent with previous in vitro results. ${ }^{22}$

The endocytosed GC-AuNPs in the cancer cells were visualized using dark-field microscopy. Without cellular uptake of GC-AuNPs, cancer cells did not show any scattered light from nanoparticles in the dark-field microscopic images ("control" in Fig. 4). If the incubation time was not long enough for endocytosis, cells did not present signals either ("1 h" in Fig. 4). By contrast, images of cells with the 3 - and 4-h cellular uptake time showed strong signals indicating the presence of nanoparticles (" 3 h" and " 4 h" in Fig. 4). In particular, more scattered light was found in the cytosol areas of the cancer cells as cellular uptake time increased. These aggregations of nanoparticles explain strong photoacoustic signals from the tissue-mimicking cell phantoms in Fig. 2. The endocytosis of GC-AuNPs was also observed

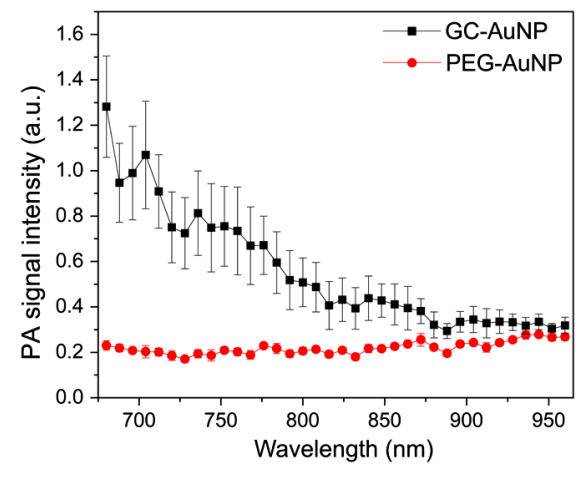

Fig. 3 Wavelength-dependent photoacoustic signal from cells incubated with PEG-AuNPs or GC-AuNPs for $4 \mathrm{~h}$. Photoacoustic signals from the GC-AuNP cells (black line) were the highest at $680 \mathrm{~nm}$ while monotonically decreasing with increased wavelength. By contrast, PEG-AuNP cells (red line) showed nearly constant photoacoustic signals regardless of excitation wavelengths. The error bars of each point indicated the standard deviation from $n=3$.

in the bright-field images, where reddish color from aggregated GC-AuNPs is notably visible. The locations of the aggregated NPs in the bright-field images are spatially correlated with the positions of the nanoparticle signals in the dark-field images. These images suggest that cellular uptake time should be at least $2 \mathrm{~h}$ for the effective endocytosis of GC-AuNPs.

\section{Discussion}

Nanoparticle synthesis and surface modification were achieved by one-step synthesis method. Because of the electronegative property, GC acted as a reducing agent for the synthesis of GC-AuNPs. ${ }^{23}$ Simultaneously, amine groups in GC interacted with the surface of AuNPs. This is why the properties of GC-AuNPs were different from that of citrate-reduced AuNPs. For example, the red-shift of SPR peak, a discrepancy of particle size between TEM and DLS, and positive zeta-potentials were observed as a result of the surface modification of AuNPs with GC. Through this simple synthesis method, we achieve a desired formulation of nanoparticles with tumor-targeting property without complex conjugation steps.

The synergistic effects of GC and AuNPs can enable the applications of GC-AuNPs in photoacoustic cancer imaging. The surface coating with GC increased the stability of GCAuNPs in physiological conditions. ${ }^{23}$ Moreover, the GC layer enhanced tumor accumulation. In the acidic condition of the 


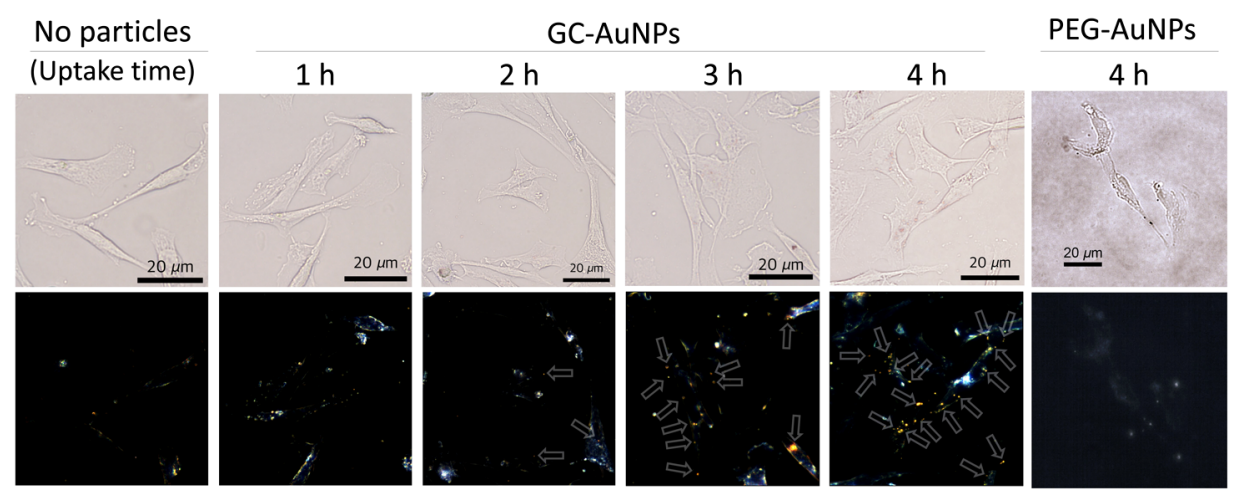

Fig. 4 The cellular uptake of GC-AuNPs in MDA-MB-231 cells. Bright-(top) and dark-field (bottom) microscopic images were taken after various cellular uptake times. In the dark-field images, arrows indicate the scattered light from GC-AuNPs. In case of PEG-AuNPs, no significant signals were detected after $4 \mathrm{~h}$ of incubation.

tumor, the amine groups of GC were decorated with more positive charges that promoted the cellular uptake of GC-AuNPs. ${ }^{24}$ These advantages of GC were combined with an optical absorption property of AuNPs, and GC-AuNP labeled cancer cells were visualized in photoacoustic imaging.

GC-AuNP cells emitted strong photoacoustic signals as evident from the photoacoustic images of tissue-mimicking cell phantoms. These strong signals originated from the accumulation of GC-AuNPs inside the cancer cells. The endocytosed GCAuNPs aggregated inside the cell and, upon laser irradiation, led to local temperature increases due to the thermal coupling of nanoparticles. This high-temperature transients temporarily increase thermal expansion coefficient which, in turn, enhanced photoacoustic signals. ${ }^{18,25}$ In contrast with GC-AuNPs, the cell incubated with PEG-AuNPs did not exhibit noticeable photoacoustic signal changes regardless of cellular uptake times. This was due to PEG molecules that impeded the cellular uptake by the inhibition of protein adsorption. ${ }^{26}$

The enhanced photoacoustic signals from the endocytosed GC-AuNPs were examined further by varying the wavelength of the pulsed laser light. As expected, the maximum photoacoustic signal intensity was observed at $680 \mathrm{~nm}$ wavelength, and then the intensity of photoacoustic signal was gradually decreasing for longer near infrared wavelengths. Such behavior was due to the aggregation of GC-AuNPs that caused the slight red-shift of optical absorption and, more importantly, a gradual decrease of optical absorption of plasmon-coupled nanoparticles. ${ }^{18,27}$ This distinct pattern of photoacoustic signal behavior can be utilized to unmix background tissue signal and signal from nanoparticles using multispectral photoacoustic imaging approach. 19,20,22,28,29 $^{2}$

The high-contrast photoacoustic images provide information complimentary to ultrasound images. Although ultrasound images can visualize structure and morphology of the imaged tissue [Fig. 2(a)], photoacoustic images [Fig. 2(b)] can be used to identify presence of GC-AuNPs and degree of cellular uptake. Overall, GC-AuNPs demonstrated their potentials as a contrast agent for cancer cell detection.

\section{Conclusion}

We have demonstrated an application of GC-AuNPs as a photoacoustic contrast agent for cancer cell imaging. GC-AuNPs showed enhanced accumulation in the cancer cells compared with PEG-AuNPs, which resulted in strong photoacoustic signals in the tissue-mimicking phantoms containing GC-AuNP labeled cancer cells. The strong photoacoustic signals originated from the aggregation of GC-AuNPs inside cancer cells confirmed by dark-field microscopy, where the scattered light from GC-AuNPs was observed in the cytosol area of cancer cells after $2 \mathrm{~h}$ of cellular uptake time. These results suggest that GCAuNPs has the potential to serve as an effective contrast agent for cancer imaging because photoacoustic imaging of cancer cells was accomplished without using antibodies or sophisticated surface modification of nanoparticles.

\section{Disclosures}

The authors declare they have no relevant financial interests.

\section{Acknowledgments}

This work was supported in part by Breast Cancer Research Foundation under Grant No. BCRF-18-043 and National Institutes of Health under Grant Nos. EB008101 and CA149740.

\section{References}

1. C. K. Kuhl et al., "Mammography, breast ultrasound, and magnetic resonance imaging for surveillance of women at high familial risk for breast cancer," J. Clin. Oncol. 23(33), 8469-8476 (2005).

2. K. Wilson, K. Homan, and S. Emelianov, "Biomedical photoacoustics beyond thermal expansion using triggered nanodroplet vaporization for contrast-enhanced imaging," Nat. Commun. 3, 618 (2012).

3. A. P. Sarvazyan et al., "Shear wave elasticity imaging: a new ultrasonic technology of medical diagnostics," Ultrasound Med. Biol. 24(9), 1419-1435 (1998).

4. S. Y. Emelianov et al., "Combined ultrasound, optoacoustic and elasticity imaging," Proc. SPIE 5320, 101-112 (2004).

5. B. Cox et al., "Quantitative spectroscopic photoacoustic imaging: a review," J. Biomed. Opt. 17(6), 061202 (2012).

6. S. Mallidi, G. P. Luke, and S. Emelianov, "Photoacoustic imaging in cancer detection, diagnosis, and treatment guidance," Trends Biotechnol. 29(5), 213-221 (2011).

7. R. Bouchard, O. Sahin, and S. Emelianov, "Ultrasound-guided photoacoustic imaging: current state and future development," IEEE Trans. Ultrason. Ferroelectr. Freq. Control 61(3), 450-466 (2014).

8. G. P. Luke, D. Yeager, and S. Y. Emelianov, "Biomedical applications of photoacoustic imaging with exogenous contrast agents," Ann. Biomed. Eng. 40(2), 422-437 (2012).

9. E. Shahriari, M. Moradi, and M. Raeisi, "An experimental study of thermal diffusivity of $\mathrm{Au}$ nanoparticles: effects of concentration particle size," J. Theor. Appl. Phys. 10(4), 259-263 (2016).

10. Y. M. Xuan, Q. Li, and W. F. Hu, "Aggregation structure and thermal conductivity of nanofluids," Aiche. J. 49(4), 1038-1043 (2003). 
11. A. Trapani et al., "The potential of glycol chitosan nanoparticles as carrier for low water soluble drugs," Int. J. Pharm. 375(1-2), 97-106 (2009).

12. J. Y. Yhee et al., "Cancer-targeted MDR-1 siRNA delivery using selfcross-linked glycol chitosan nanoparticles to overcome drug resistance," J. Control. Rel. 198, 1-9 (2015).

13. J. H. Na et al., "Real-time and non-invasive optical imaging of tumortargeting glycol chitosan nanoparticles in various tumor models," Biomaterials 32(22), 5252-5261 (2011).

14. J. H. Kim et al., "Hydrophobically modified glycol chitosan nanoparticles as carriers for paclitaxel (Reprinted from Journal of Controlled Release, vol 109, pg 1, 2005)," J. Control. Rel. 111(1-2), 228-234 (2006).

15. I. C. Sun et al., "Tumor-targeting gold particles for dual computed tomography/optical cancer imaging," Angew. Chem. Int. Edit. 50(40), 9348-9351 (2011).

16. I. C. Sun et al., "Biocompatible glycol chitosan-coated gold nanoparticles for tumor-targeting CT imaging," Pharm. Res. 31(6), 1418-1425 (2014).

17. E. Chung et al., "Evaluation of gold nanotracers to track adipose-derived stem cells in a PEGylated fibrin gel for dermal tissue engineering applications," Int. J. Nanomed. 8, 325-336 (2013).

18. S. Y. Nam et al., "Nonlinear photoacoustic signal increase from endocytosis of gold nanoparticles," Opt. Lett. 37(22), 4708-4710 (2012).

19. S. Mallidi et al., "Visualization of molecular composition and functionality of cancer cells using nanoparticle-augmented ultrasound-guided photoacoustics," Photoacoustics 3(1), 26-34 (2015).

20. G. P. Luke et al., "Sentinel lymph node biopsy revisited: ultrasoundguided photoacoustic detection of micrometastases using molecularly targeted plasmonic nanosensors," Cancer Res 74(19), 5397-5408 (2014).

21. J. R. Cook, R. R. Bouchard, and S. Y. Emelianov, "Tissue-mimicking phantoms for photoacoustic and ultrasonic imaging," Biomed. Opt. Express 2(11), 3193-3206 (2011).

22. S. Mallidi et al., "Multiwavelength photoacoustic imaging and plasmon resonance coupling of gold nanoparticles for selective detection of cancer," Nano Lett. 9(8), 2825-2831 (2009).

23. D. R. Bhumkar et al., "Chitosan reduced gold nanoparticles as novel carriers for transmucosal delivery of insulin," Pharm. Res. 24(8), 1415-1426 (2007).

24. S. H. Crayton and A. Tsourkas, "pH-titratable superparamagnetic iron oxide for improved nanoparticle accumulation in acidic tumor microenvironments," ACS Nano 5(12), 9592-9601 (2011).
25. C. L. Bayer et al., "Photoacoustic signal amplification through plasmonic nanoparticle aggregation," J. Biomed. Opt. 18(1), 016001 (2013).

26. C. D. Walkey et al., "Nanoparticle size and surface chemistry determine serum protein adsorption and macrophage uptake," J. Am. Chem. Soc. 134(4), 2139-2147 (2012).

27. S. Y. Nam et al., "In vivo ultrasound and photoacoustic monitoring of mesenchymal stem cells labeled with gold nanotracers," PloS One 7(5), e37267 (2012).

28. T. Hirasawa et al., "Spectral-differential-based unmixing for multispectral photoacoustic imaging," Appl. Opt. 57(10), 2383-2393 (2018).

29. B. Wang et al., "Plasmonic intravascular photoacoustic imaging for detection of macrophages in atherosclerotic plaques," Nano Lett. 9(6), 2212-2217 (2009).

In-Cheol Sun obtained a $\mathrm{PhD}$ in biomedical engineering from Georgia Institute of Technology and Emory University is a postdoctoral fellow in the Ultrasound Imaging and Therapeutics Research Laboratory in the School of Electrical and Computer Engineering at Georgia Tech. His research focuses on development of nanoagents for imaging and image-guided therapy using ultrasound and photoacoustic imaging.

Cheol-Hee Ahn is a professor in the Department of Materials Science and Engineering, Seoul National University, and director of NanoBio Materials Laboratory. His research area is in the development of polymeric gene delivery carriers, nanomaterials, and cell delivery systems for curing cancer and real-time molecular imaging.

Kwangmeyung Kim is principal researcher at Korea Institute of Science and Technology (KIST). His research interests are non-invasive and diagnostic imaging for various human diseases, molecular and cellular imaging for biological processes, and inorganic/organic nanoparticle-based imaging probes.

Stanislav Emelianov is a Joseph M. Pettit Endowed Chair, Georgia Research Alliance Eminent Scholar, and professor of electrical and computer engineering and biomedical engineering at the Georgia Institute of Technology and Emory University School of Medicine. Furthermore, he is director of the Ultrasound Imaging and Therapeutics Research Laboratory. Projects in Dr. Emelianov's laboratory are focused on the discovery, development, and clinical translation of diagnostic imaging and therapeutic instrumentation, augmented with theranostic nanoagents. 